\title{
DERIVAÇÃO VENTRICULOPERITONEAL COM VÁLVULA NO TRATAMENTO DA HIDROCEFALIA DO LACTENTE
}

\author{
Gilberto Machado de Almeida * \\ Walter C. Pereira *
}

Nos últimos anos as derivações ventriculovenosas têm sido largamente empregadas no tratamento da hidrocefalia. Contudo, algumas complicações graves, especialmente relacionadas com processos infecciosos ou trombembólicos, fizeram arrefecer o entusiasmo por essas técnicas. Estudos anátomo-patológicos demonstraram a alta incidência de microembolismo e fibrose pulmonares nos pacientes submetidos a derivações ventriculovenosas ${ }^{2,3}, 4,7,8,9,10$. Se bem que manifestações clínicas de cor pulmonale crônico sejam raramente observadas $1,2,4$ é possível que, com o passar dos anos, surjam conseqüências graves.

Por outro lado, as derivações para o peritônio geralmente funcionavam durante pouco tempo devido ao bloqueio da extremidade inferior do cateter, sendo necessárias revisões frequentes. Entretanto, adaptando-se uma válvula unidirecional ao sistema de drenagem ventriculoperitoneal a extremidade distal do mesmo se mantém sempre cheia de líquido cefalorraqueano, dificultando sua oclusão.

\section{MATERIAL E TÉCNICA}

De dezembro de 1965 até março de 1969 usamos derivação ventriculoperitoneal com válvula em 110 doentes, 31 dos quais apresentavam neurocisticercose ou tumores da linha média e não serăo aqui considerados. Estudaremos apenas 79 pacientes com hidrocefalia.

Entre as 79 crianças, 12 tinham sido submetidas, antes da derivação para o peritônio, a ventrículo-atriostomias ou revisões, num total de 33 intervenções cirúrgicas.

A técnica empregada para a derivacão ventriculoperitoneal não apresenta particularidade alguma. A extremidade proximal do sistema é instalada da mesma maneira que na derivação ventriculo-atrial, deixando-se 30 ou $40 \mathrm{~cm}$ do cateter distal de silástico livres na cavidade peritoneal. Foram empregadas válvulas de Holter, Pudenz e Hakin, conforme a tabela seguinte:

Departamento de Neurologia da Faculdade de Medicina da Universidade de São Paulo (Prof. Adherbal Tolosa): * Neurocirurgiões. 
1. operação Revisões Total

\begin{tabular}{llccc}
\hline \hline Holter de pressão média & 62 & 5 & 67 \\
Holter de pressão baixa & 10 & - & 10 \\
Pudenz de pressão média & 1 & 1 & 2 \\
Hakin de pressão média & 5 & 5 & 2 \\
Hakin de pressão baixa & 1 & 7 & 86 \\
\hline
\end{tabular}

Reoperaçóes foram praticadas 24 vêzes, visando à: retirada do sistema de drenagem por infecção (10); retirada por penetração do cateter no intestino grosso (1); nova derivação após retirada do sistema (4); revisão do cateter ventricular (2); revisão do cateter peritoneal (2); substituição da válvula por drenagem insuficiente (3); tratamento de hematoma subdural (2).

\section{R E S U L T A O S}

Não nos foi possivel acompanhar a evolução da hidrocefalia em 14 crianças. Das 65 restantes, 59 estão bem, sem hipertensão intracraniana, 5 faleceram e uma passa mal, com quadro de sofrimento grave do tronco cerebral, causado por hematoma subdural bilateral, que se instalou após a derivação ventriculoperitoneal com válvula de Hakin de pressão baixa.

Julgamos interessante assinalar que, nas duas crianças nas quais se desenvolveu hematoma subdural, haviam sido usadas válvulas de Hakin (pressão baixa em uma e média na outra). E possivel que a maior capacidade de drenagem do líquido cefalorraqueano tenha favorecido a formação dos hematomas por colapso cerebral intenso.

A válvula continua funcionando bem em 53 das 59 crianças que estão com a hidrocefalia compensada. Nas demais ou o sistema de drenagem foi retirado (6 casos) ou não está funcionando satisfatòriamente (1 caso). Êstes dados contrariam a opinião defendida por Foltz ${ }^{5,6}$ de que a derivação, uma vez instalada, é necessária para tôda a vida do paciente.

A obstrução do cateter distal, ocorrência comum nas técnicas clássicas de drenagem (sem válvula) para o peritônio, foi encontrada apenas duas vêzes. As infecçōes foram a principal causa de complicação, sendo responsáveis pelo óbito das 5 crianças. Parece-nos, entretanto, que nas drenagens para o peritônio o problema infeccioso é mais fàcilmente controlado que nas derivaçōes ventriculovenosas. Realmente, em nenhuma ocasião surgiu quadro de peritonite grave, tendo o óbito ocorrido por causa da meningencefalite.

\section{R E S M O}

As derivaçōes ventriculovenosas, se bem que tenham propiciado grande progresso no tratamento da hidrocefalia, não podem ser consideradas como solução definitiva do problema. Entre os vários inconvenientes que surgiram com estas técnicas, o embolismo e a fibrose pulmonares avultam entre os 
mais sérios, sendo suas conseqüências tardias ainda desconhecidas. Julgamos, portanto, justificada a busca de novas técnicas cirúrgicas ou o aprimoramento de outras já conhecidas.

De dezembro de 1965 a março de 1969 empregamos a derivação ventriculoperitoneal com válvula em 110 doentes, 79 dos quais eram crianças com hidrocefalia. Nossos resultados permitiram verificar que, do ponto de vista mecânico, as derivações para a corrente sangüinea e para o peritônio funcionam de maneira semelhante, não sendo necessárias revisões freqüentes, como ocorria quando eram feitas drenagens para o peritônio sem válvula. Por outro lado, com as derivações ventriculoperitoneais evitamos a microembolia pulmonar; além disso as infecções no sistema de drenagem provocam quadro menos grave e de mais fácil solução que o observado nas ventrículo-atriostomias.

\section{S U M M A R Y}

Ventriculo-peritoneal shunt with valve in the treatment of hydrocephalus.

In spite of being a significant advance in the treatment of hydrocephalus, ventriculo-venous shunts cannot be considered a definitive solution. Among several disadvantages which have ariven from the widespread use of such techniques pulmonary embolism and fibrosis should be emphasized, the late consequences of which are unknown as yet. The search for new techniques and/or the improvement of old procedures thus seem quite justified.

During the last four years we have employed a ventriculo-peritoneal shunt with valve in 110 patients, including 79 hydrocephalic children. The results have shown that physically both the valve shunts draining into the peritoneum or into the blood stream work similarly. In both instances reoperations have not been frequent, as it was the case when no valves were used in the peritoneal shunts. On the other hand, by using ventriculo-peritoneal shunts not only pulmonary microembolism is prevented but also infections involving the draining system are less severe and more easily controlled than those occurring in the ventriculo-atrial shunts.

\section{REFER N CIAS}

1. ALMEIDA, G. M. - Derivação ventrículo-atrial no tratamento da hidrocefalia em crianças. Arq. Neuro-Psiquiat. (São Paulo) 27:1, 1969.

2. CROME, L. \& ERDOHAZI, M. - Main pathological findings in hydrocephalic children treated by ventriculo-atrial shunt. Arch. Dis. Child. 41:179, 1966.

3. EMERY, J. L. \& HILTON, H. B. - Lung and heart complications of the treatment of hydrocephalus by ventriculo-auriculostomy. Surgery 50:309, 1961.

4. ERDOhAZI, M.; ECKSTEIN, H. V. \& CROME, L. - Pulmonary embolization as a complication of ventriculo-atrial shunts inserted for hydrocephalus. Develop. Med. Child. Neurol. (Suppl.) 11:36, 1966. 
5. FOLTZ, E. L. - The first seven years of a hydrocephalus project. In J. Shulman: Workshop in Hydrocephalus. University of Pennsylvania, Philadelphia, 1965 , p. 79.

6. FOLTZ, E. L. \& SHURTLEFF, D. B. - Five year comparative study of hydrocephalus in children with and without operation (113 cases). J. Neurosurg. 20:1064, 1963.

7. FRIEDMAN, S.; SITA-GOZUM, C. \& CHATTEN, J. - Pulmonary vascular changes complicating ventriculo-vascular shunting for hydrocephalus. J. Pediat. $64: 305,1964$.

8. NUGENT, C. R.; JUDY, M.; BLOOR, B. M. \& WARDEN, H. - Thromboembolic complications of ventriculo-atrial shunts. J. Neurosurg. 24:34, 1966.

9. SPERLING, D. R.; PATRICK, J, R.; ANDERSON, F. N. \& FYLER, D. C. Cor pulmonale secondary to ventriculo-auriculostomy. Amer. J. Dis. Child. 107:308, 1964.

10. TALNER, N. S.; OBERMAN, H. A.; SCHMIDT, R. W. - Thromboembolism complicating Holter valve shunt. Amer. J. Dis. Child. 101:602, 1961.

Departamento de Neurologia - Faculdade de Medicina da Universidade de São Paulo - Caixa Postal 3461 - São Paulo, SP - Brasil. 